\title{
Activation of an endogenous retrovirus-associated long non-coding RNA in human adenocarcinoma
}

Ewan A Gibb ${ }^{1,2^{*}}$, René L Warren ${ }^{1}$, Gavin W Wilson ${ }^{3,4}$, Scott D Brown ${ }^{1,5}$, Gordon A Robertson ${ }^{1}$, Gregg B Morin ${ }^{1,2,6+}$ and Robert A Holt ${ }^{1,2,6+}$

\begin{abstract}
Background: Long non-coding RNAs (IncRNAs) are emerging as molecules that significantly impact many cellular processes and have been associated with almost every human cancer. Compared to protein-coding genes, IncRNA genes are often associated with transposable elements, particularly with endogenous retroviral elements (ERVs). ERVs can have potentially deleterious effects on genome structure and function, so these elements are typically silenced in normal somatic tissues, albeit with varying efficiency. The aberrant regulation of ERVs associated with InCRNAs (ERV-IncRNAs), coupled with the diverse range of IncRNA functions, creates significant potential for ERV-IncRNAs to impact cancer biology.

Methods: We used RNA-seq analysis to identify and profile the expression of a novel IncRNA in six large cohorts, including over 7,500 samples from The Cancer Genome Atlas (TCGA).

Results: We identified the tumor-specific expression of a novel IncRNA that we have named Endogenous retroViral-associated ADenocarcinoma RNA or 'EVADR', by analyzing RNA-seq data derived from colorectal tumors and matched normal control tissues. Subsequent analysis of TCGA RNA-seq data revealed the striking association of EVADR with adenocarcinomas, which are tumors of glandular origin. Moderate to high levels of EVADR were detected in 25 to $53 \%$ of colon, rectal, lung, pancreas and stomach adenocarcinomas (mean $=30$ to 144 FPKM), and EVADR expression correlated with decreased patient survival (Cox regression; hazard ratio $=1.47$, $95 \%$ confidence interval $=1.06$ to 2.04, $P=0.02$ ). In tumor sites of non-glandular origin, EVADR expression was detectable at only very low levels and in less than $10 \%$ of patients. For EVADR, a MER48 ERV element provides an active promoter to drive its transcription. Genome-wide, MER48 insertions are associated with nine IncRNAs, but none of the MER48-associated IncRNAs other than EVADR were consistently expressed in adenocarcinomas, demonstrating the specific activation of EVADR. The sequence and structure of the EVADR locus is highly conserved among Old World monkeys and apes but not New World monkeys or prosimians, where the MER48 insertion is absent. Conservation of the EVADR locus suggests a functional role for this novel IncRNA in humans and our closest primate relatives.
\end{abstract}

Conclusions: Our results describe the specific activation of a highly conserved ERV-InCRNA in numerous cancers of glandular origin, a finding with diagnostic, prognostic and therapeutic implications.

\footnotetext{
* Correspondence: egibb@bcgsc.ca

${ }^{\dagger}$ Equal contributors

'Genome Sciences Centre, British Columbia Cancer Agency, 675 West 10th

Ave, Vancouver, British Columbia V5Z 1L3, Canada

${ }^{2}$ Department of Medical Genetics, University of British Columbia, Vancouver,

British Columbia V6T 1Z4, Canada

Full list of author information is available at the end of the article
} 


\section{Background}

The mammalian transcriptome is pervasively transcribed [1-4]. Comprehensive transcriptome sequencing surveys have revealed many classes of widely expressed non-coding RNAs, including long non-coding RNAs (lncRNAs) [5]. These novel genes encode mRNA-like transcripts that are by definition at least 200 nucleotides in length and have no apparent protein coding capacity, but are otherwise subject to normal mRNA processing, including 5 ' capping, splicing and polyadenylation [6]. Many lncRNAs demonstrate exquisite cellular-, tissue- or developmental stage-specific expression patterns [7-9]. IncRNAs have a range of demonstrated functions, including chromatin remodeling [10], alternative splicing [11] and mRNA degradation [12], and their dysregulation has been linked to many disorders, including cancer [13-15].

Compared with protein-coding genes, lncRNA genes tend to associate with transposable elements, particularly with endogenous retroviruses (ERVs) [16-18]. The majority of ERVs are genomic relics of exogenous retrovirus insertions which, although typically degenerated, may retain active promoter and polyadenylation signals encoded within their flanking long terminal repeats (LTRs) [19,20]. Through these active regulatory elements, both ERVs and other retrotransposons contribute significantly to the regulation of gene expression [21,22]. However, unregulated ERV LTRs can promote aberrant transcription and are therefore typically silenced in adult tissues by epigenetic mechanisms including, but not limited to, DNA methylation [23,24]. In cancer, silenced ERVs may be released from normal cellular regulation, resulting in a general increase in ERV-mediated transcription [25-27]. Given the frequency and scattered genomic distribution of ERVs and the influence of their LTRs on the expression of neighboring genes, ERVs have high potential to promote oncogene expression or to alter host gene expression networks to favor tumor development [25]. Similarly, changes in physiological or cellular conditions that promote classspecific ERV LTR expression could promote the activation of lncRNAs associated with that class, as has been observed for HERV-H LTRs in embryonic stem cells $[18,28]$. Considering the range of biological functions described for lncRNAs and their role in gene regulation, ERV-mediated lncRNA activation has considerable potential to impact cellular biology.

To further characterize the relationship between aberrant lncRNA expression and cancer, we evaluated RNA-seq data from colorectal adenocarcinoma and matched normal control tissues from 65 subjects for lncRNA expression. We identified a 394-nucleotide MER48 LTR ERV-associated lncRNA 'EVADR' as being robustly expressed in tumor samples, with virtually no expression in corresponding normal tissues. We then profiled the EVADR lncRNA across 25 different cancer types, determined the promoter activity of the MER48 LTR in vitro, mapped the genomewide MER48 LTR expression, and surveyed the conservation of the EVADR gene locus across 13 primates. Here we report the identification and initial characterization of $E V A D R$.

\section{Methods}

Cancer, normal tissue and cell line transcriptome datasets All sequence files were obtained with permission and stored in a secure file system. The sequence data used in this study are listed in Table 1.

\section{Ethics}

The research described herein conformed to the Helsinki Declaration. All clinical specimens were obtained previously [33] with informed consent by the BC Cancer Agency Tumor Tissue Repository (BCCA-TTR), which operates as a dedicated biobank with approval from the University of British Columbia-British Columbia Cancer Agency Research Ethics Board (BCCA REB; certificate \#H09-01268).

\section{RNA-seq read mapping}

Raw sequence reads were aligned to the human reference genome and transcriptome (hg19, Ensembl v.70) using STAR v.2.3.0e [34] or TopHat v.2.0.6 [35]. STAR was run with the following parameters: minimum/maximum intron size set to 30 and 500,000, respectively, noncanonical, unannotated junctions were removed, maximum tolerated mismatches was set to 10 , and the outSAMstrandField intronMotif option was enabled. For TopHat reads aligned to human ribosomal RNA sequences $(18 \mathrm{~S}, 28 \mathrm{~S}, 5 \mathrm{~S})$ were removed, and the remaining reads were aligned to human gene annotations (hg19, Ensembl v.70) using default command line options except the minimum isoform fraction option was set to 0 . The Cuffdiff command included with Cufflinks v.2.0.2 [36] was used to calculate the fragments per kilobase of exon per million fragments mapped (FPKM) with upper quartile normalization, fragment bias correction, and multiread correction enabled. All other options were set to default.

\section{Targeted read assembly (TASR)}

Targeted read assembly was performed using TASR v.1.5.1 with the following parameters enabled: $-\mathrm{m} 20-\mathrm{o}$ 2 -i 1 [37]. Briefly, the 397-nucleotide EVADR cDNA sequence (ENST00000418403) was used as the target for TASR, which extracted overlapping $k$-mers (-k 15) from this sequence as seeds. These were used to recruit EVADR RNA-seq reads from The Cancer Genome Atlas (TCGA) bam files, and the set of reads recruited from each sample was independently assembled de novo. Sequence contigs of 200 nucleotides and larger were aligned to the EVADR 
Table 1 RNA-seq datasets used in this study

\begin{tabular}{|c|c|c|c|}
\hline Server & Dataset ID & Description & Download host \\
\hline SRA & SRP010181 & Colorectal cancer tumor matched normal libraries $(n=130)$ & [29] \\
\hline CGhub & Various & Twenty-five different cancers and normal tissues $(n=7,677)$ from TCGA & [30] \\
\hline GEO & GSE40419 & Lung adenocarcinoma tumor matched normal libraries $(n=144)$ & [31] \\
\hline GEO & GSE30611 & Human Bodymap 2.0 representing 16 normal human tissues & [31] \\
\hline ENCODE & Various & Fasta files from 15 different cell lines, replicates downloaded where available & [32] \\
\hline SRA & SRP008280 & Triplicate control K562 cell RNA-seq & [29] \\
\hline
\end{tabular}

GEO, Gene Expression Omnibus; SRA, Sequence Read Archive; TCGA The Cancer Genome Atlas.

reference sequence with BLAST (v.2.2.22; parameters -a 8 -F F -p blastn -m 7), keeping only assembled transcripts with $90 \%$ or higher sequence identity [38]. The number of sequence reads assembled was tallied in each TCGA sample and RPK (reads per kilobase) and RPKMS (reads per kilobase per million sequenced) values were calculated [39].

\section{RT-PCR}

RNA and cDNA were generated as described [40]. In this study, cDNA from tumor or normal tissues was used as a template for PCR using primers oHL_0005 and oHL_0006 (Table 2). The reaction was performed for 35 cycles with Taq DNA polymerase (NEB, Ontario, Canada) in supplied buffer and amplicons were resolved on a $1 \%$ agarose gel.

\section{Cell culture}

K562 and SW480 cells were cultured in Dulbecco's modified Eagle's medium (DMEM) supplemented with $10 \%$ fetal bovine serum and penicillin (100 units $/ \mathrm{ml}$ )streptomycin $(100 \mu \mathrm{g} / \mathrm{ml})$ (Life Technologies, Ontario, Canada). Cells were maintained at $37^{\circ} \mathrm{C}$ in a $5 \% \mathrm{CO}_{2}$ incubator.

\section{Plasmids}

The MER48 promoter deletion plasmid constructs were generated by first amplifying the MER48 element specific to EVADR using primers oHL_0011 and oHL_0005.
The subsequent amplicon was gel purified, diluted 1:100 and used as a template for amplifying each truncated version of the MER48 element: MER1F, oHL_0011 and oHL_0013; MER2F, oHL_0012 and oHL_0013; MER3F, oHL_0015 and oHL_0013; and MER_FLIP, oHL_0016 and oHL_0013. Amplicons were digested with XhoI and HindlII, gel purified and ligated into the pGL4 vector (Promega, Wisconsin, United States).

\section{Reporter assays}

K562 cells were suspended in Electroporation Buffer (20 mM HEPES, $137 \mathrm{mM} \mathrm{NaCl}, 5 \mathrm{mM} \mathrm{KCl}, 0.7 \mathrm{mM}$ $\mathrm{Na}_{2} \mathrm{HPO}_{4}, 6 \mathrm{mM}$ dextrose, $50 \mathrm{mM}$ trehalose, 1\% DMSO) with $30 \mu \mathrm{g}$ plasmid and electroporated at $200 \mathrm{~V}, 975 \mu \mathrm{F}$ capacitance. SW480 cells were grown to $60 \%$ confluency and then reverse transfected using Lipofectamine 2000 (Life Technologies, Ontario, Canada) according to the manufacturer's instructions. The cells were harvested $24 \mathrm{~h}$ after electroporation (for K562) or $48 \mathrm{~h}$ after transfection (for SW480), and firefly and Renilla luciferase activities were analyzed with the Dual-Luciferase ${ }^{\circledR}$ Reporter Assay System, according to the manufacturer's instructions (Promega, Wisconsin, United States). All experiments were repeated in triplicate.

RNA ligase-mediated rapid amplification of cDNA ends 5' Rapid amplification of cDNA ends (RACE) was performed with a RLM-RACE kit (Life Technologies, Ontario Canada) according to the manufacturer's instructions

Table 2 Primer sequences

\begin{tabular}{lll}
\hline Oligo & Purpose & Sequence (5'-3') \\
\hline OHL_0005 & Forward RT-PCR primers for EVADR transcript & TGATGCCATTTCAGCCTCAG \\
OHL_0006 & Reverse RT-PCR primers for EVADR transcript & TGGCCGCTCAGATTCTCTATC \\
OHL_0011 & Clone MER48 LTR upstream of EVADR. Xhol/HindIII & GGCTCGAGTAAGGGAATGAATAACTCCG \\
OHL_0012 & Clone MER48 LTR upstream of EVADR. Xhol/HindIII & GGCTCGAGATATGTACCCTGTGAAGACC \\
OHL_0015 & Cloning small fragment of MER48 element & GGCTCGAGGTGCTGATGCCATIITCAGCC \\
OHL_0013 & Clone MER48 LTR upstream of EVADR. Xhol/HindIII & GGAACCTTACATGCTGTTITAATGAGCG \\
OHL_0016 & Same as oHL_0011 but reversed restriction site. Xhol & CCAAGGTTAAGGGAATGAATAACTCCG \\
OHL_0017 & Same as oHL_0012 but reversed restriction site. HindIII & GGCTCGAGACATGCTGTTTAATGAGCG \\
OHL_0014 & Same primer as oHL_0006 but for cloning 5'RACE products & CCGGATCCTGGCCGCTCAGATTCTCTATC \\
\hline
\end{tabular}


using total RNA isolated from K562 cells and the reverse primer oHL_0014.

\section{MER48 expression patterns from K562 cell line data} A list of 201 reliable MER48 coordinates was obtained from Dfam [41]. This list was used to count reads aligning to MER48 elements from two replicate K562 transcriptome .bam files obtained from ENCODE using readcount v0.01 with all options set to default.

\section{Statistical tests}

All statistical tests were performed using R v.3.1.0.

\section{Tumor and normal expression comparisons}

For each of the colorectal and lung cancer datasets, we compared the difference between mean tumour expression values and mean normal tissue expression values using a two-sample paired $t$-test.

\section{STAR/Cufflinks and TASR expression correlations}

The Pearson correlation coefficient was used to quantify the strength of the correlations between the STAR/Cufflinksand TASR-generated expression levels.

\section{EVADR tumor site association}

All patients for each cancer type were classified according to whether EVADR was detectable ( $>0$ RPKMS). Next, we counted the total number of patients in the two groups (adenocarcinoma and non-adenocarcinoma), with and without detectable EVADR expression. Using these counts, we performed a Chi-squared test to test the significance of the observed association of EVADR expression and adenocarcinoma.

\section{Reporter assays}

The dual luciferase promoter mapping data were subjected to $t$-tests with unequal variance followed by Bonferroni correction, comparing the mean relative luciferase units (RLU) of the MER1F construct with the RLUs of each of the MER2F, MER3F and MER_FLIP constructs, in each of the K562 and SW480 cell lines.

\section{Correlation of MER48-IncRNA expression}

For each cancer type we performed an ANOVA test to test the null hypothesis that the expression of all MER48lncRNAs were the same. A significant result from ANOVA was obtained for each of the five cancer types, which prompted a subsequent Tukey's honest significant difference post hoc test to identify which MER48-IncRNAs were differentially expressed in each cancer type.

\section{Structural conservation}

Using the program Molecular Evolutionary Genetics Analysis (MEGA) v.6 [42], we inferred the evolutionary history of the primates using the maximum parsimony method and EVADR cDNA alignments. The resultant tree (Figure $S 1$ in Additional file 1) was used to count the number of nucleotide changes in context of EVADR evolution in primates. To test the differences in the number of variable positions in base-paired sequences (structured regions) compared with non-structured sequences, we used a Chi-squared test to compare the count of sites in structured and non-structured sequence classes lacking perfect sequence conservation across all studied species. To test whether the observed nucleotide substitutions in structured regions were more likely to maintain base pairing than expected by chance, we used a Chi-squared test to compare the observed distribution with the distribution expected by random substitutions. To create this null distribution, we tallied for GC, GU and AU base pairs all possible random nucleotide substitutions (eight per pair, including single nucleotide deletions). Of the 24 possible combinations, four nucleotides are expected to still base pair after random mutation.

\section{Sequence identity calculations}

Genomic alignments were generated using ClustalW and imported into Jalview2 [43] for manual curation. The polished alignments were exported as a ClustalW alignment file and uploaded to the SIAS [44] website to determine the sequence identity of EVADR between primates. We included parameters to take gaps into account and used the BLOSUM62 matrix; all other settings were set to default. The tree was generated using the UCSC genome tool phlyoGif $[45,46]$.

\section{Coding potential analysis}

To confirm that EVADR was non-coding, we used the online coding potential calculator tool (CPC) to calculate EVADR's coding potential [47]. As a positive control, we included the cancer-associated lncRNA UCA1. The results of this analysis were both transcripts were considered to be non-coding, with a CPC score of -1.29 for $E V A D R$ and -1.14 for $U C A 1$, where values between -1 and 1 are marked as 'weak non-coding' or 'weak coding', respectively. Non-coding RNAs score below -1 and protein coding genes score above 1 .

Using the online tool ORF Finder [48], we identified three putative open reading frames (ORFs) within EVADR, including one ORF with a non-AUG start codon (Figure S2 in Additional file 1). To determine whether these ORFs were actively translated, we queried several K562 proteomic datasets from studies that specifically evaluated the presence of small peptides and found no evidence for translation of the EVADR ORFs [49-51]. These findings further support the classification of EVADR as a lncRNA with little to no apparent translation. 


\section{Heatmaps}

The MER48-lncRNA heatmaps were clustered using centroid linkage and Euclidean distance in custom Python scripts.

\section{Genome mining MER48-associated IncRNAs}

Dfam was used to identify MER48 elements within $500 \mathrm{bp}$ or $50 \mathrm{bp}$ of a predicted lncRNA transcriptional start site using Ensembl v.70 annotations. We identified nine candidates using $500 \mathrm{bp}$ and eight using $50 \mathrm{bp}$; in both cases $E V A D R$ was included. Manual examination of the excluded lncRNA ENSG00000265374 revealed the MER48 formed part of the exon of the IncRNA, but the transcriptional start site was upstream of the repeat element. We included this lncRNA in our analyses.

\section{Results}

Identification of a highly upregulated long non-coding RNA in colorectal carcinoma

To identify lncRNAs associated with colorectal cancer, we performed whole transcriptome sequence analysis on 65 tumor and matched normal colorectal poly(A)selected RNA-seq libraries generated in a previously described study [33]. From these data we identified two Ensembl-curated yet uncharacterized lncRNAs, ENSG00000222041 and ENSG00000237643, which were strongly upregulated in colorectal tumors compared with matched normal control tissues. We did not characterize ENSG00000222041 further because its differential expression was less substantial than that of ENSG00000237643, and because it had a complex alternative splicing pattern that complicated further analysis. Further scrutiny of ENSG00000237643 (which we named EVADR for Endogenous retroViral-associated ADenocarcinoma RNA) revealed significantly increased expression of this lncRNA in tumors $(14.4 \pm 24.5$ FPKM (mean \pm standard deviation (SD))) compared with matched normal control tissues, where it was typically not expressed $(0.50 \pm 1.52$ FPKM $($ mean $\pm \mathrm{SD})$ ) (Figure 1a; $P=2.8 \mathrm{e}-05$; $t$-test). Genomic analysis revealed a predicted 397-nucleotide lncRNA with three exons and a single transcript isoform located on chromosome 6, $91.8 \mathrm{~kb}$ downstream of Col9A1 and $13.5 \mathrm{~kb}$ upstream of FAM135A (Figure 1b). Despite high expression of EVADR in tumor samples, we did not observe differential expression of genes flanking $E V A D R$ (Figure 1b). We designed primers to amplify a $200 \mathrm{bp}$ region of the mature EVADR transcript and validated the expression of this lncRNA in the colorectal tumor and normal tissues using RT-PCR (Figure 1c,d; Additional file 2). To corroborate our observation that EVADR was not expressed in non-malignant tissue, we measured EVADR expression in 16 normal adult human tissues finding only weak expression in lung and prostate tissues (Figure S3A in Additional file 1). Expanding these analyses to 15 cell lines, we found that EVADR was highly expressed (122 FPKM) in the chronic myeloid leukemia K562 cell line, but its expression was extremely low $(<1$ FPKM) in the others, including in the H1-HESC embryonic stem cell line (Figure S3B in Additional file 1), where it was 0.04 FPKM. These data demonstrate that EVADR is selectively expressed.

\section{Pan-cancer analysis reveals high activation of EVADR in human adenocarcinoma}

The striking expression pattern observed for EVADR in colorectal carcinoma prompted us to perform additional analysis across a broad panel of human cancers. We queried EVADR expression in all available tumor $(\mathrm{n}=$ $7,043)$ and normal $(\mathrm{n}=634)$ RNA-seq libraries from TCGA Research Network project. Because full transcriptome assembly requires significant compute resources we opted to analyze TCGA RNA-seq data specifically for EVADR expression using the targeted de novo assembler TASR [37], which is much faster in situations where transcriptome-wide information is not required. To convert TASR-derived assemblies to expression values, we tallied assembled sequence reads and calculated the RPKMS. To determine the concordance between Cufflinks-derived FPKMs and TASR-derived RPKMS we compared EVADR expression across 181 colon adenocarcinoma (COAD) samples and observed a Pearson correlation of $r=0.93$. Next, we used TASR to quantify EVADR expression in 7,677 tumor and normal TCGA RNA-seq datasets (Figure 2a). Consistent with our initial observation that EVADR was specifically upregulated in colorectal tumors, we found that this lncRNA was detected in TCGA colon and rectal tumor datasets at high (mean 25.3 \pm 22.7 RPKMS (mean \pm SD)) expression levels (Figure 2a,b). Strikingly, we also found high EVADR expression in numerous lung (LUAD), pancreatic (PAAD), and stomach (STAD) adenocarcinomas $(12.3 \pm 13.8,12.1 \pm 17.2$, and $3.7 \pm 7.7$ RPKMS, respectively; Figure 2a,b; Figure S4 in Additional file 1). We found that while 481 out of 1,223 adenocarcinomas expressed $E V A D R$ at detectable levels, only 50 out of 5,289 nonadenocarcinomas expressed EVADR. We performed a Pearson's Chi-squared test comparing these two groups and found that EVADR was significantly $(P<2.2 \mathrm{e}-16)$ associated with adenocarcinomas, but not with the other tumor types. Having confirmed high EVADR expression in the colorectal tumors in two independent datasets, we also validated the cancer association of EVADR in a noncolorectal tumor and matched normal control tissue dataset. We processed an independent lung adenocarcinoma transcriptome dataset [52] using Cufflinks [36], and observed high EVADR expression in the tumors $(9.84 \pm 24.7$ FPKM (mean \pm SD)) and weak or undetectable expression in normal tissues (mean $0.44 \pm 0.55$ FPKM (mean \pm SD)) $(P=0.002 ; t$-test; Figure S5 in Additional file 1$)$. These 


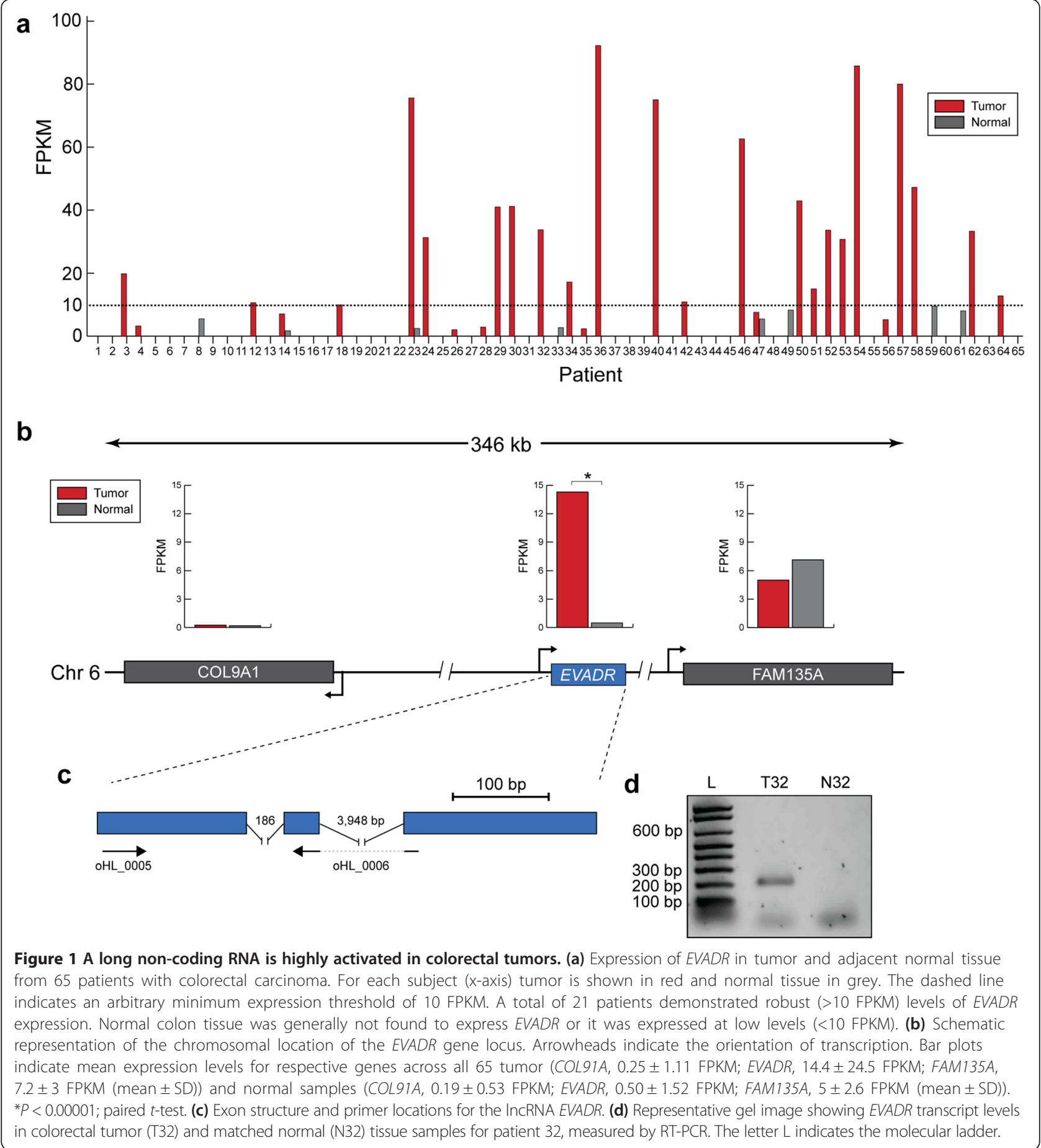

data were consistent with the high EVADR expression in lung adenocarcinoma observed in the TCGA samples.

\section{Clinical features of EVADR track with poor prognosis in adenocarcinomas}

Having observed specific and high-level expression of EVADR in the tumors of patients with lung, colon, rectal, stomach and pancreatic adenocarcinoma, we sought to identify any possible clinical relevance of the association of EVADR with these tumor types. To explore this possibility, we selected TCGA datasets for these five adenocarcinoma tumor types because of their large patient cohorts and the availability of corresponding clinical data. Using a Cox proportional-hazard model that accounted for known prognostic factors (age, gender, type of adenocarcinoma, and tumor stage) as well as EVADR expression 

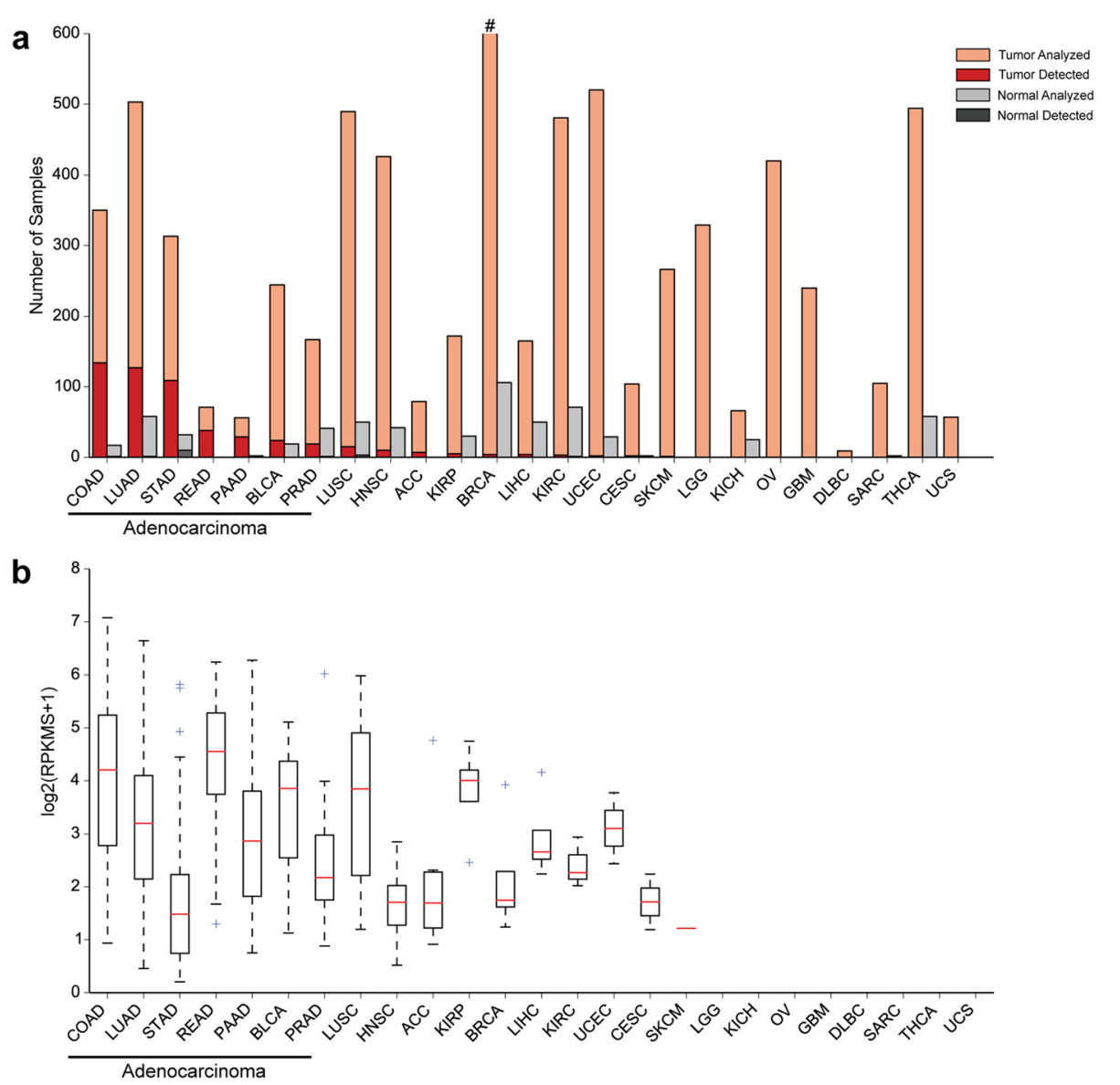

Figure 2 EVADR is robustly expressed in adenocarcinomas. (a) EVADR expression in 25 TCGA cancer types and corresponding normal tissues. Light orange indicates the tumors analyzed and light grey indicates normal samples analyzed. The hashtag (\#) indicates that 916 BRCA samples were analyzed. Dark red indicates the number of adenocarcinoma samples in which EVADR expression was detected, while dark grey indicates the number of normal samples in which EVADR was detected. (b) EVADR expression as $\log _{2}(\mathrm{RPKMS}+1)$, determined for tumors using TASR. Medians are indicated by red lines, upper and lower quartiles by the boxes, and outliers by blue crosses. COAD, colon adenocarcinoma; LUAD, lung adenocarcinoma; STAD, stomach adenocarcinoma; READ, rectum adenocarcinoma; PAAD, pancreatic adenocarcinoma; BLCA, bladder urothelial carcinoma; PRAD, prostate adenocarcinoma; LUSC, lung squamous cell carcinoma; HNSC, head and neck squamous cell carcinoma; ACC, adrenocortical carcinoma; KIRP, kidney renal papillary cell carcinoma; BRCA, breast invasive carcinoma; LIHC, liver hepatocellular carcinoma; KIRC, kidney renal clear cell carcinoma; UCEC, uterine corpus endometrial carcinoma; CESC, cervical squamous cell carcinoma and endocervical adenocarcinoma; SKCM, skin cutaneous melanoma; LGG, brain lower grade glioma; KICH, kidney chromophobe; OV, ovarian serous cystadenocarcinoma; GBM, glioblastoma multiforme; DLBC, lymphoid neoplasm diffuse large B-cell lymphoma; SARC, sarcoma; THCA, thyroid carcinoma; UCS, uterine carcinosarcoma.

( $>5.6$ FPKM; threshold determined using Cutoff Finder [53]), we saw a significantly $(P<0.02)$ decreased overall survival for patients with high EVADR expression (Figure 3). These data associate elevated EVADR expression with lower overall survival for the five TCGA tumor types investigated.

\section{An ERV LTR contributes a functional promoter to EVADR}

Detailed sequence analysis of the EVADR genomic locus revealed sequence identity with a MER48 LTR, which is an endogenous retroviral element of the ERV1 family (Figure 4a) [54]. The MER48 LTR contributes 127 nucleotides to the primary sequence of the $5^{\prime}$ exon of EVADR, and also encodes numerous transcription factor binding sites and a putative TATA box, suggesting a possible role for these regulatory sequences in the transcriptional activation of EVADR in adenocarcinoma. As $\mathrm{K} 562$ cells strongly express $E V A D R$, we selected these cells for $5^{\prime}$ RACE [55] and mapped three distinct $5^{\prime}$ transcript termini to the EVADR MER48 LTR, each downstream of the predicted TATA box (Figure $4 \mathrm{~b}$ ). These data refine the length of the predominant EVADR transcript from the predicted value of 397 nucleotides to the confirmed value of 394 nucleotides. To experimentally test the capacity of the MER48 LTR in driving downstream transcription, we generated a series of truncated MER48 constructs and measured promoter activity, in triplicate, using a dual 


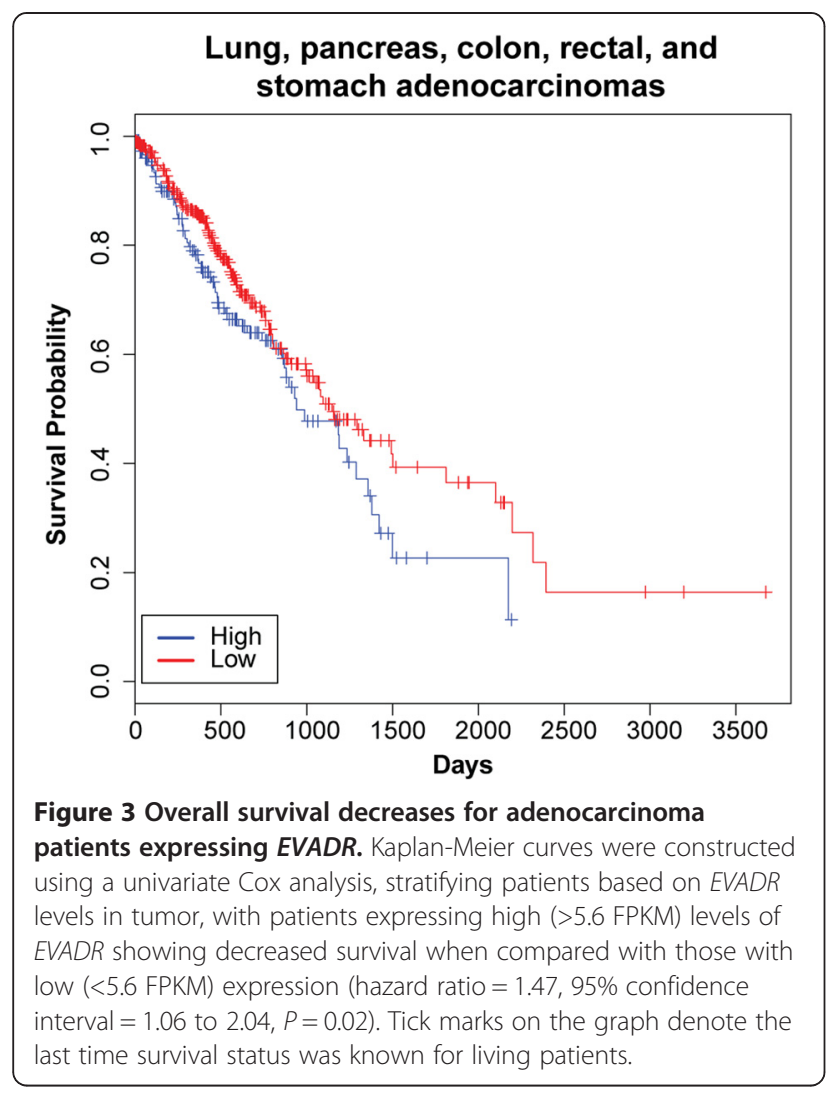

luciferase assay (Figure 4c). While full-length MER48 is active in both K562 and SW480 cells (MER1F; K562, $73.7 \pm 5.1$ RLU (mean \pm SD); SW480 8.14 \pm 0.40 RLU), each subsequent $5^{\prime}$ truncation dramatically reduced luciferase activity (MER2F; K562, mean $1.45 \pm 0.14$ RLU; SW480, $1.26 \pm 0.08 \mathrm{RLU})$, with negligible promoter activity observed for MER48 sequence overlapping with the $5^{\prime}$ exon of EVADR (MER3F; K562, $0.08 \pm 0.01$ RLU; SW480, $0.10 \pm 0.01 \mathrm{RLU}$ ) (Figure 4d,e). Surprisingly, we observed strong luciferase activity when the EVADR MER48 LTR was in the opposite orientation (MER_FLIP; K562, $198 \pm$ 15.4 RLU; SW480, 23.6 \pm 2.4 RLU), indicating the MER48 LTR can function as a bidirectional promoter (Figure 4d,e; MER_FLIP). Despite the high transcriptional activity observed from the MER_FLIP plasmid constructs, we found no evidence supporting bidirectional transcription in vivo (Figure S6 in Additional file 1). These results demonstrate that the MER48 LTR not only contributes to the $5^{\prime}$ exon of $E V A D R$, but also provides a promoter sequence capable of driving the transcription of this IncRNA.

\section{EVADR is selectively upregulated in adenocarcinomas}

A MER48 LTR is found within $500 \mathrm{bp}$ of the annotated transcriptional start sites of nine Ensembl annotated lncRNAs. To determine if EVADR expression is due to a general activation of MER48 LTR elements in adenocarcinomas, we queried the expression of the other eight
non-EVADR MER48-associated lncRNAs in colon, rectal, pancreas, stomach and lung tumors using Cufflinks [36]. These tumor types were selected because they included large numbers of patients that expressed EVADR at high levels. EVADR clustered independently of other MER48lncRNAs and of tumor type (Figure 5a). Next, we determined the distribution of IncRNA expression for all MER48-lncRNAs in each of the five different types of adenocarcinoma, and for each type we observed significantly elevated expression of EVADR relative to all other MER48-IncRNAs $(P<2 \mathrm{e}-16$ for each cancer type; ANOVA with Tukey's honest significant difference post hoc test; Figure 5b). Additionally, in lung adenocarcinoma, ENSG00000231106 is also expressed differently from all other MER48-lncRNAs (including EVADR). Finally, to determine whether MER48 LTRs were universally active, we queried the expression of 201 Dfam-curated MER48 LTRs in K562 cells and found that the MER48 LTR associated with $E V A D R$ is specifically and highly activated, while the remaining MER48 elements were inactive or showed only minimal expression (Figure 6a). To validate these data, we examined an additional K562 RNA-seq dataset to quantify the MER48-lncRNAs using Cufflinks [36] (Figure 6b). The highest expressed MER48-lncRNA in this dataset was EVADR (230 FPKM), consistent with the read countderived data in Figure 6a. The lncRNA ENSG00000230257 also showed moderately increased expression (21 FPKM) in K562 cells, while the expression of the other seven lncRNAs was low or undetectable (Figure 6b). Collectively, these results demonstrate that EVADR is specifically activated and not due to global MER48 activation.

\section{EVADR is a highly conserved, primate-specific IncRNA}

Comparative sequence analysis of the EVADR MER48 LTR revealed high (>90\%) sequence identity to the EVADR MER48 LTR in Old World monkeys (OWMs) and apes. Interestingly, however, the EVADR LTR is absent in more distant primates, including New World monkeys (NWMs) and prosimians (Figure 7a,b). The full 394 bp EVADR lncRNA gene demonstrated high sequence identity in apes and OWMs, particularly in the exons, with the introns showing decreasing identity with increasing phylogenetic distance (Figure 7b; Additional file 3). Both EVADR introns (i1 and i2) retained conserved GT/AG splice junctions in the apes and OWMs, but these junctions were not consistently conserved in NWMs or prosimians. The high nucleotide conservation for EVADR corresponds to an evolutionarily conserved secondary structure in OWMs and apes (Figure 8), with significantly more variable nucleotide positions occurring in unpaired or loop positions (47/152) than in structured regions $(39 / 242$ of paired nucleotide positions) $(P=0.0008$; Chi-squared test; Figures S7 and S8 in Additional file 1). The 39 substitutions in structured regions affected a total 


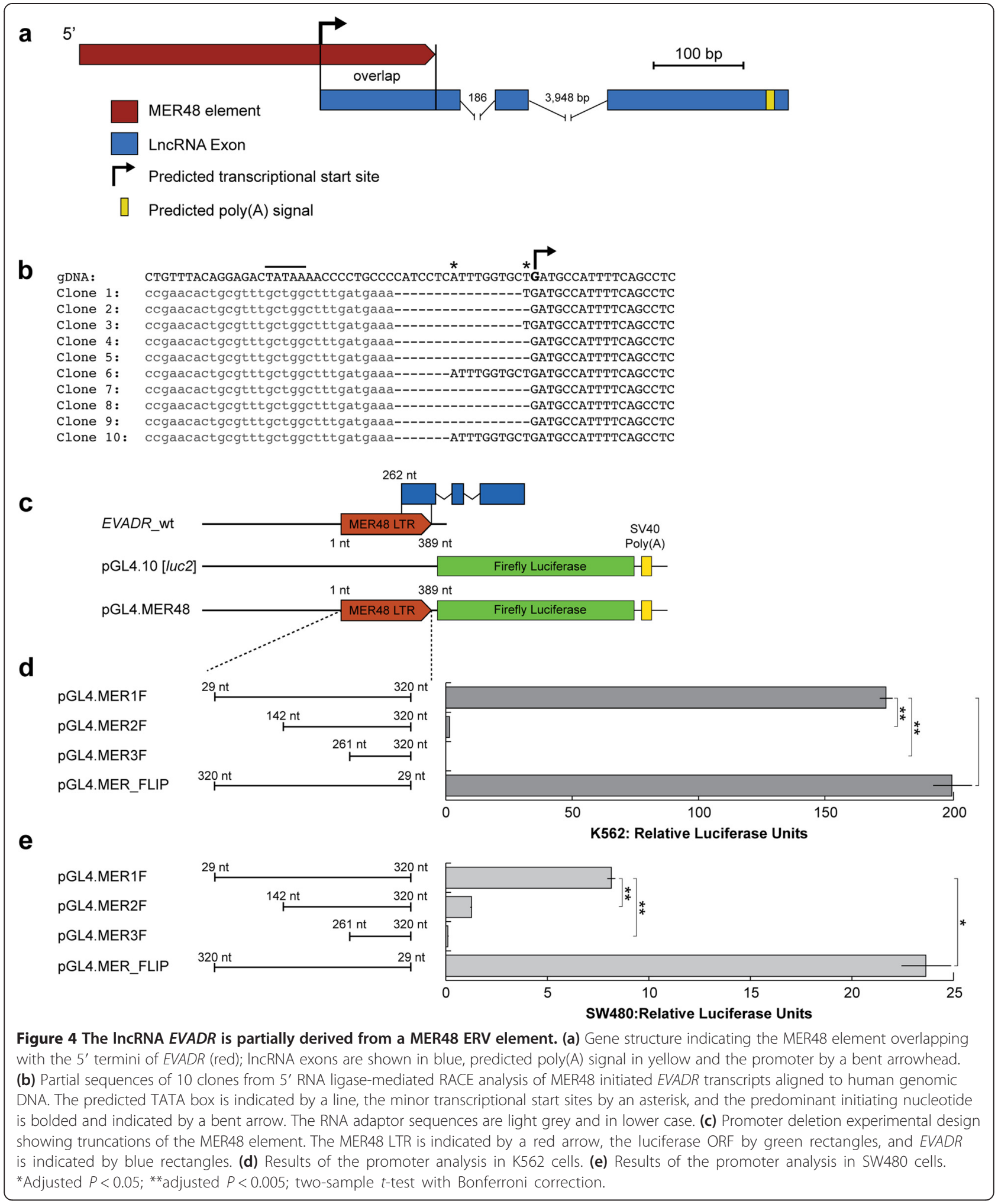

of 32 base pairs at 27 locations, where 19 out of 39 substitutions were compensatory, maintaining base pairing and 20 out of 39 were non-compensatory, disrupting base pairing (Figure S8 in Additional file 1). Given 4 out of 24 random possible nucleotide changes in $\mathrm{GC}, \mathrm{GU}$ and $\mathrm{AU}$ base pairs would maintain base pairing, we find that nucleotide substitutions in stems are significantly more likely to be compensatory (19/39 versus $7 / 39$; observed versus 
a

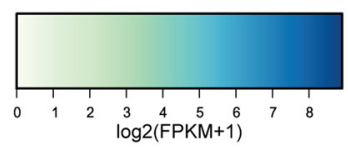

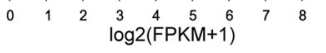
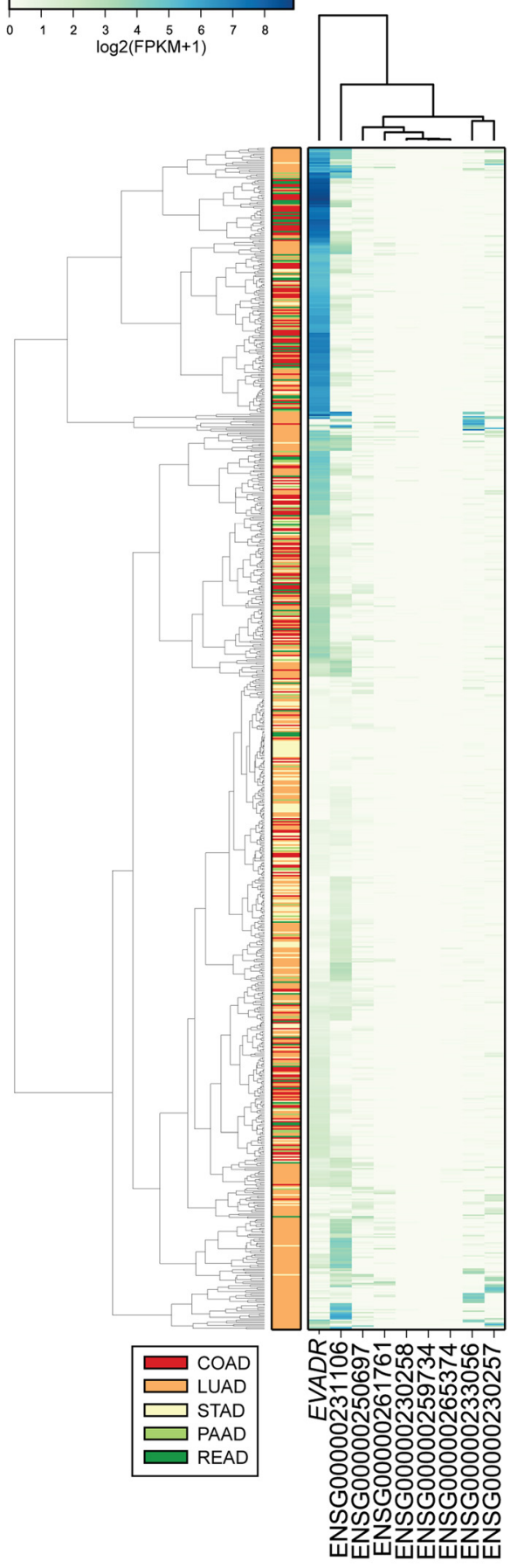

b
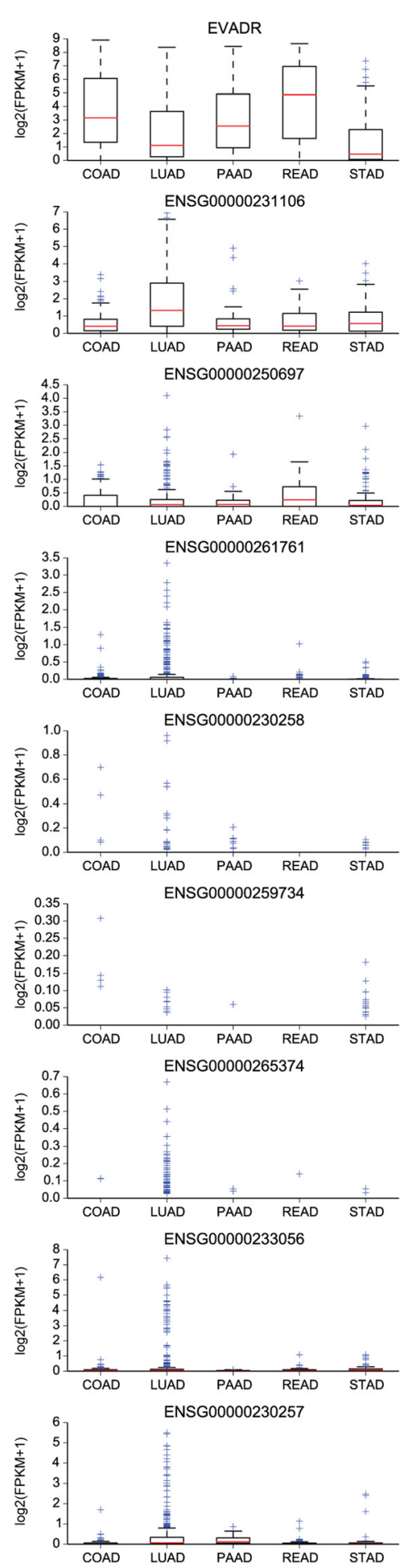

Figure 5 MER48 activation is specific, rather than general, in adenocarcinoma tumors. (a) Clustered heatmap showing the expression of nine MER48-associated IncRNAs in a panel of colon (COAD; $n=181$ ), rectal (READ; $n=66)$, pancreatic (PAAD; $n=52)$, lung (LUAD; $n=372)$ and stomach (STAD; $n=136$ ) adenocarcinomas. (b) Expression levels for the same nine MER48-IncRNAs. The $y$-axis is $\log _{2}(F P K M+1)$ for each set. Medians are indicated by red lines, upper and lower quartiles by boxes, and outliers by blue crosses. 


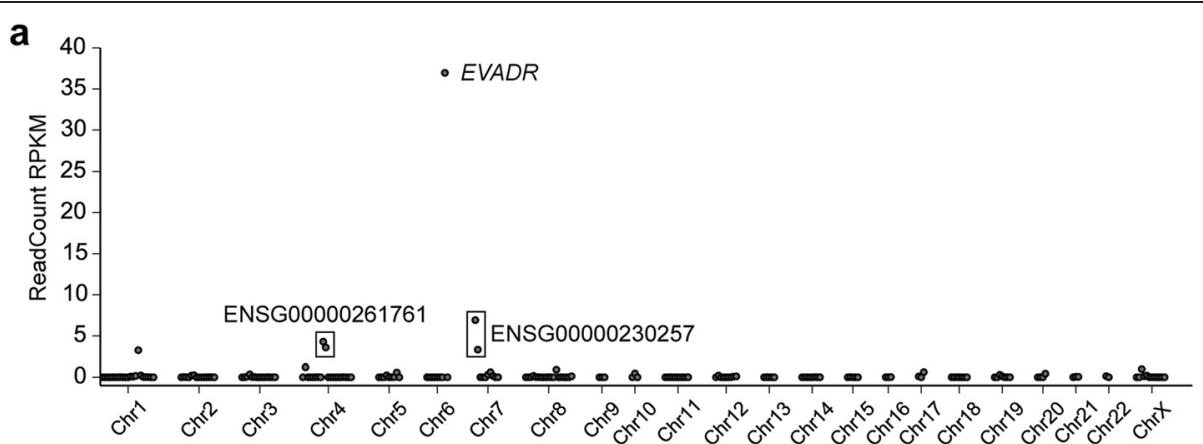

\section{b}

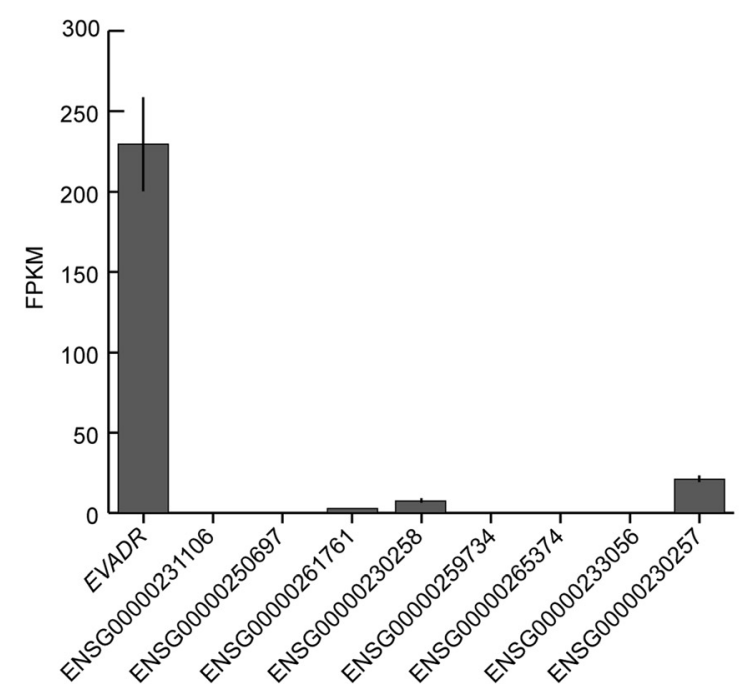

Figure 6 MER48 LTRs are not globally active in K562 cells. (a) Scatterplot of expression for 201 reliable MER48 elements in K562, with EVADR being the highest expressed MER48 element. Plotted values are the average of two experiments. (b) Expression of the nine MER48-IncRNAs in K562s in a validation dataset. Values are the average of three experiments. The ENSG00000230257 is driven by a MER48 element flanking a HERVH48 insertion. The ENSG00000261761 IncRNA MER48 is split by an Alu insertion. The IncRNA ENSG00000230258 is associated with an unreliable MER48 in Dfam [41] and was not, therefore, part of the list used for the scatterplot and does not appear in these data.

expected) than non-compensatory (20/39 versus $33 / 39$; observed versus expected) base pairs in stem regions $(P=$ 7.8e-08, Chi-squared test). These results show the EVADR lncRNA has strong conservation in primates closest to humans, at both the primary and secondary structural levels.

\section{Discussion}

We report the tumor-specific activation of EVADR, a novel lncRNA gene which is associated with a MER48 LTR. This lncRNA demonstrates nominal to low expression in normal tissue, but is significantly upregulated in cancer, particularly in colon, rectal, lung, stomach and pancreas adenocarcinomas.

Approximately $8 \%$ of the human genome is composed of ERV elements [58-60], with a number of ERV1, ERVLMaLR, ERVL and ERVK elements overlapping a substantial number of lncRNAs [18]. The MER48 LTR is a member of the ERV1 class of retroviral elements, and is likely derived from an unknown retrovirus distantly related to RTVL-H2 [54]. Like other ERV elements, the MER48 LTRs are prominent in the human genome with over 200 non-redundant insertions [41], overlapping nine different lncRNA genes (this study). With at least one MER48 repeat found inserted into an Alu-J element, the MER48 repeat can be dated to at most 80 million years before present [54,61], which is at least 10 million years after the estimated time of divergence of rodents and primates [62]. In apes and OWMs, the MER48 LTR and corresponding EVADR lncRNA are highly conserved. While MER48 elements are present in NWMs and prosimians we could not identify a MER48 insertion at the expected EVADR locus in these groups (Figure 7). These observations suggest an active MER48 element inserted at the EVADR locus sometime after the split between NWMs and OWMs, but before the split between OWM and apes. As the MER48 LTR provides a promoter and contributes sequence to the EVADR exons, it appears the apes and 
a
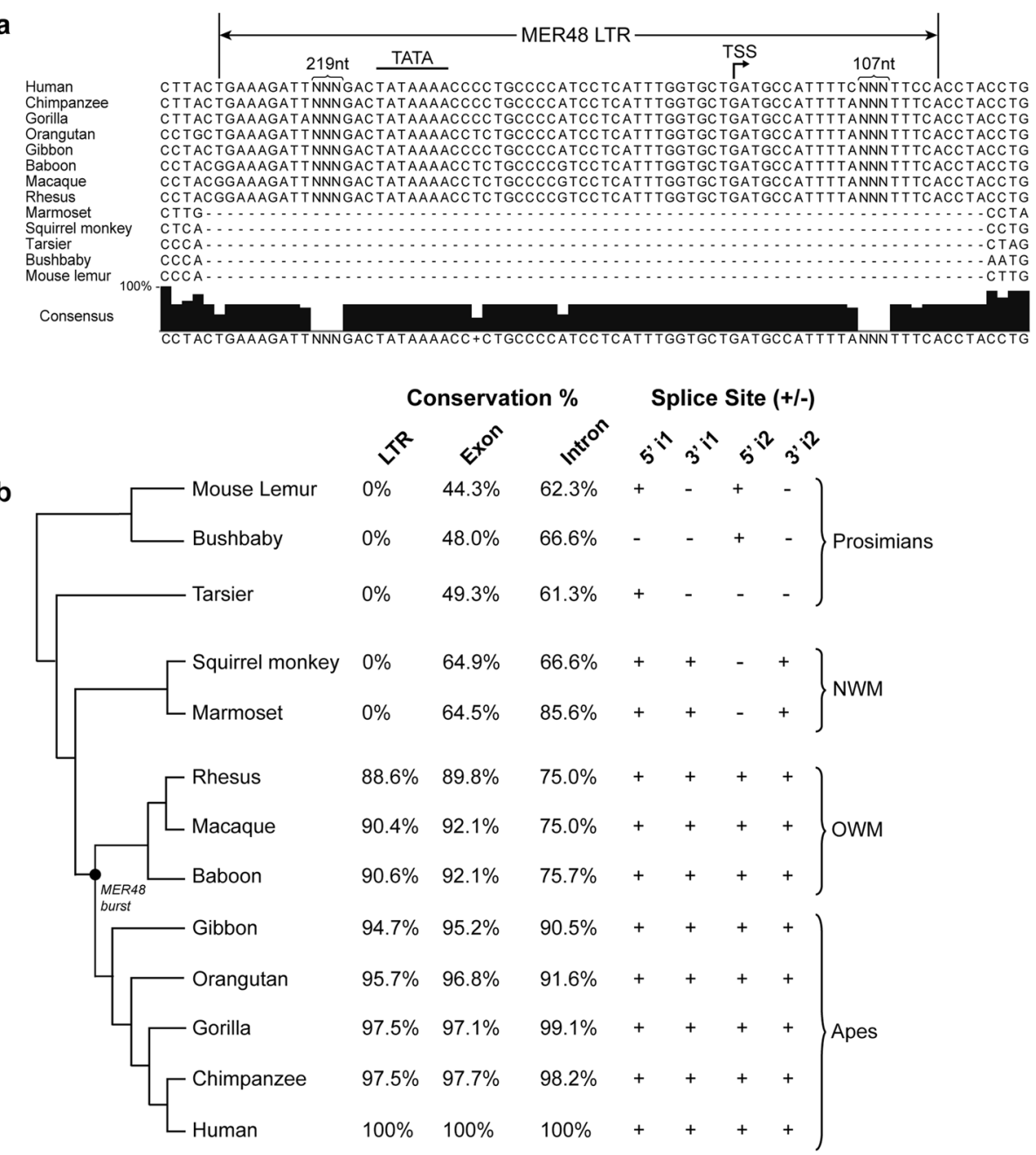

Figure 7 Sequence conservation of the EVADR IncRNA in primates. (a) Partial sequence alignment of the EVADR MER48 and flanking sequence in 13 primates showing lack of MER48 LTR in NWMs and prosimians. The major experimentally determined transcriptional start site (TSS) is indicated by a bent arrow, while the predicted TATA box is indicated by a line. Due to space constraints, some sequence has been removed and is indicated by NNN and curly brackets. (b) Sequence identity of the EVADR MER48 LTR and EVADR in 13 primate species determined with SIAS (Methods) on ClustalW aligned sequences. The first and second introns are indicated by an i1 and i2, respectively. The tree was generated using the UCSC genome tool phlyoGif $[45,46]$. The black dot indicates a burst of MER48 insertion as determined by the GEnome-wide Browser for RETroelement (GEBRET) webtool [56]. For GEBRET output and complete sequence alignments see Figure S9 in Additional file 1 and ClustalW alignments in Additional file 3, respectively.

OWMs have co-opted a MER48 insertion to generate the lncRNA EVADR and this arrangement has been maintained evolutionarily.

Unlike most lncRNAs, which show modest conservation [5,63-65], the human EVADR lncRNA has remarkably high sequence identity among non-human primates, similar to the constrained exonic sequences of protein coding genes. Analysis of the EVADR consensus structure reveals the majority of the evolutionary structural base pair substitutions are compensatory, which strongly suggests the RNA secondary structure is critical for $E V A D R$ function (Figure 8). Further supporting strong purifying selection for $E V A D R$, we find the splice junctions of both introns to be conserved in apes and OWMs, but not in NWMs or prosimians. These findings are consistent with previous reports describing conserved splice junctions for lncRNAs $[66,67]$. Many lncRNAs do not have high sequence or structural conservation and yet can have biological functions $[64,68,69]$. However, the high level of sequence conservation observed for $E V A D R$ is atypical; this suggests the biological function of EVADR may also be manifested through its RNA structural features rather than its primary sequence. Collectively, the constraint on the EVADR exons, splice junctions and promoter sequence, coupled 


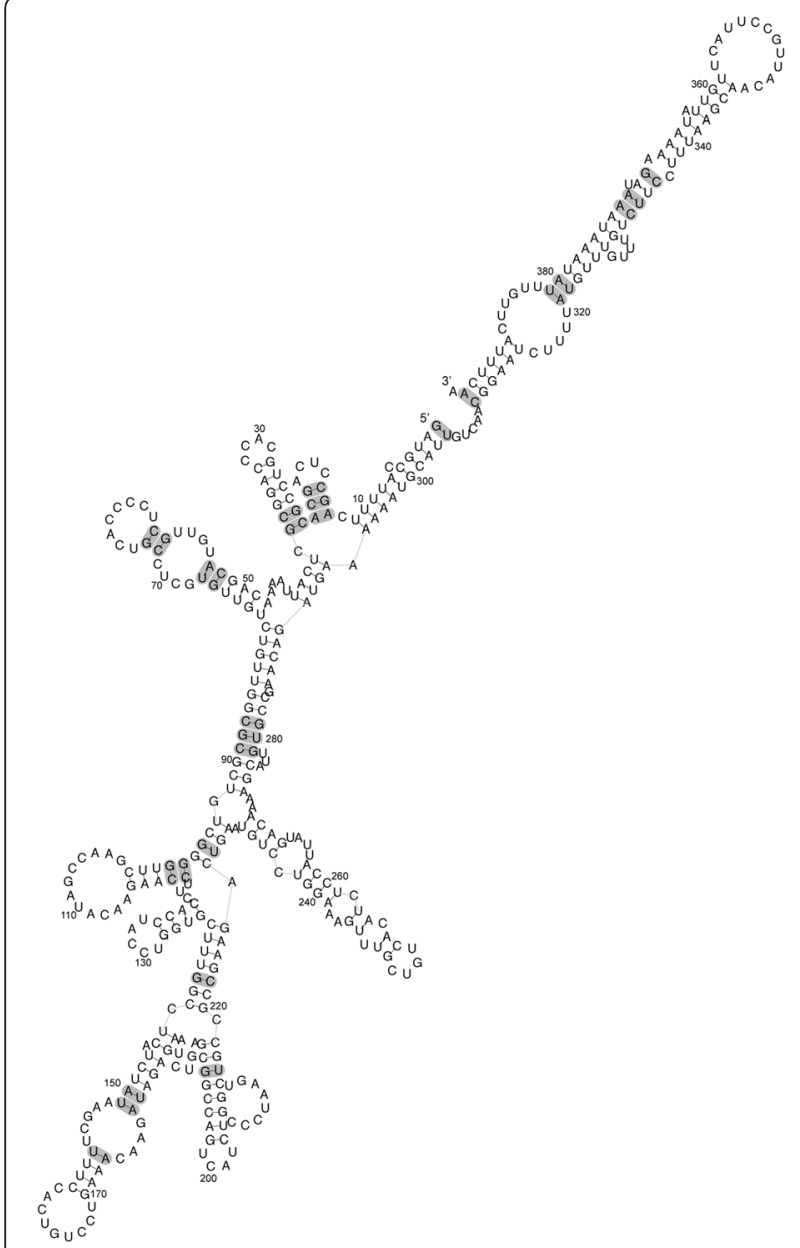

Figure 8 Human EVADR secondary structure. RNAalifold was used to predict a secondary structure based on a consensus fold for apes and OWMs [57]. Shading indicates the 27 base pair positions with nucleotide substitutions. The EVADR alignments showing all nucleotide substitutions and a chart showing only the base pair substitutions are shown in Figures S7 and S8 in Additional file 1, respectively.

with the restricted expression patterns, suggest this gene may play an important, yet undetermined role in primate biology.

The EVADR MER48 LTR not only contributes sequence to the $5^{\prime}$ exon of the lncRNA, but also a functional promoter that may direct aberrant expression of EVADR in cancer. We quantified EVADR expression across 25 different TCGA tumor types to find this lncRNA is strongly associated with colon, rectal, lung, stomach and pancreas adenocarcinomas, but not with corresponding normal tissues. In general, the eight other MER48-lncRNAs have low expression in these same tumors and do not correlate with EVADR tumor expression profiles. Thus, relative to other MER48-lncRNAs, there is specific activation of $E V A D R$ in these adenocarcinomas. There is a possibility that $E V A D R$ has a non-adenylated form which would not be detectable in TCGA datasets since they are derived from poly(A)+ RNA. Two other studies have found ERV LTRmediated activation of gene expression in cancer but both of these studies described protein coding genes that employ an ERV LTR as an alternative promoter. The first study reported activation of the colony stimulating factor 1 receptor $(C S F 1 R)$ gene in Hodgkin's lymphoma [70] and the second reported activation of the fatty acid binding protein 7 (FABP7) gene in diffuse large B-cell lymphoma [71]. In cancer cell lines there are a number of examples of the recently described very long intergenic non-coding RNAs (vlincRNAs) displaying transcriptional activation mediated by a ERV [72]. While it is known that ERV LTRs can drive aberrant expression of protein-coding genes, our study expands this to show they can modulate the expression of lncRNAs in human cancer in a highly specific manner.

The adenocarcinoma-specific expression of EVADR suggests this lncRNA may have a distinct function in tissues and tumors of a glandular origin. Consistent with this observation, many lncRNAs have been reported to demonstrate tissue- or cell type-specific expression [7,8]. More recently, another class of ERV-associated IncRNAs, HERVH-lncRNAs, have been reported to be specifically active in embryonic stem cells, where they are associated with pluripotency $[18,73,74]$. However, HERV-H LTRs also demonstrate stem cell-specific expression [28], which may suggest the HERVH-lncRNAs are activated, at least in part, by virtue of their association with the HERVLTR. We found no evidence for EVADR expression in stem cells in our analysis of ENCODE cell line RNA-seq data (Figure S3B in Additional file 1), but it is possible $E V A D R$ may be active in early developmental processes. In a manner similar to the HERVH-lncRNAs, the MER48 LTRs may provide a regulatory mechanism to drive tissueor tumor-specific lncRNA expression.

\section{Conclusions}

The conservation of EVADR in recent primate evolution and the MER48-mediated activation of EVADR in adenocarcinoma highlight the need for further studies to elucidate the normal function of $E V A D R$ and its relevance to cancer biology. Ongoing experiments will identify $E V A D R$ 's protein and RNA interacting partners, chromatin binding sites, and effects on gene expression, and EVADR knock-in mice will likely be useful for elucidating $E V A D R$ 's biological and oncogenic phenotypes. It is also possible that the activation of EVADR does not contribute to oncogenesis, but rather is a consequence of changes to the transcriptional regulatory environment typical of cancer cells. Regardless of a biological function, the specificity of EVADR activation in adenocarcinomas coupled with the poorer survival probability that tracks with elevated EVADR expression suggest that further characterization of $E V A D R$ as a candidate adenocarcinoma biomarker is warranted. 


\section{Additional files}

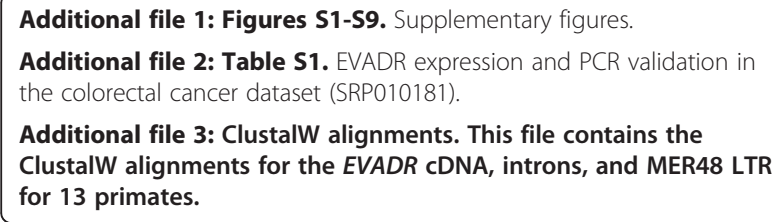

\section{Abbreviations}

ERV: endogenous retrovirus; EVADR: Endogenous retroViral-associated ADenocarcinoma RNA; FPKM: fragments per kilobase of exon per million fragments mapped; IncRNA: long non-coding RNA; LTR: long terminal repeat; NWM: New World monkey; ORF: open reading frame; OWM: Old World monkey; RACE: rapid amplification of CDNA ends; RLU: relative luciferase units; RPK: reads per kilobase; RPKMS: reads per kilobase per million sequenced; SD: standard deviation.

\section{Competing interests}

The authors declare that they have no competing interests.

\section{Authors' contributions}

EAG conceived the study, designed and performed the computational analysis and laboratory experiments, and wrote the manuscript. RLW performed computational analysis and contributed to drafting of the manuscript. GWW performed computational analysis and contributed to figures. SDB performed statistical and computational analyses. AGR performed computational analysis. GBM and RAH provided oversight and scientific direction for the study and preparation of the manuscript. GBM and RAH contributed equally. All authors have read and approved the manuscript for publication

\section{Acknowledgements}

We acknowledge Georg Hausner for assistance with phylogenetic analysis and Payal Sipahimalani for project management support. The results published here are in part based upon data generated by The Cancer Genome Atlas managed by the NCl and NHGRI. Information about TCGA can be found at http://cancergenome.nih.gov. Further, we acknowledge the ENCODE Consortium for providing the cell line datasets used within. This work was supported by a BC Proteomics Network (BCPN) grant to EAG, RAH and GBM. EAG was supported by postdoctoral fellowships from the Canadian Institutes of Health Research (CIHR) and the Michael Smith Foundation for Health Research (MSFHR).

\section{Author details}

${ }^{1}$ Genome Sciences Centre, British Columbia Cancer Agency, 675 West 10th Ave, Vancouver, British Columbia V5Z 1L3, Canada. ${ }^{2}$ Department of Medical Genetics, University of British Columbia, Vancouver, British Columbia V6T 1Z4, Canada. ${ }^{3}$ Informatics and Biocomputing Platform, Ontario Institute for Cancer Research, Toronto, Ontario M5G 0A3, Canada. ${ }^{4}$ Department of Molecular Genetics, University of Toronto, Toronto, Ontario M5S 1A8, Canada. ${ }^{5}$ Genome Science and Technology Program, University of British Columbia, Vancouver, British Columbia V6T 1Z4, Canada. ${ }^{6}$ Department of Molecular Biology and Biochemistry, Simon Fraser University, Burnaby, British Columbia V5A 1S6, Canada.

Received: 3 December 2014 Accepted: 12 February 2015

Published online: 05 March 2015

\section{References}

1. Djebali S, Davis CA, Merkel A, Dobin A, Lassmann T, Mortazavi A, et al. Landscape of transcription in human cells. Nature. 2012;489:101-8.

2. Carninci P, Kasukawa T, Katayama S, Gough J, Frith MC, Maeda N, et al. The transcriptional landscape of the mammalian genome. Science. 2005:309:1559-63.

3. Kapranov P, St Laurent G, Raz T, Ozsolak F, Reynolds CP, Sorensen PH, et al. The majority of total nuclear-encoded non-ribosomal RNA in a human cell is 'dark matter' un-annotated RNA. BMC Biol. 2010;8:149.
4. Kapranov P, Cheng J, Dike S, Nix DA, Duttagupta R, Willingham AT, et al. RNA maps reveal new RNA classes and a possible function for pervasive transcription. Science. 2007;316:1484-8.

5. Derrien $T$, Johnson R, Bussotti G, Tanzer A, Djebali S, Tilgner $H$, et al. The GENCODE v7 catalog of human long noncoding RNAs: analysis of their gene structure, evolution, and expression. Genome Res. 2012;22:1775-89.

6. Mercer TR, Dinger ME, Mattick JS. Long non-coding RNAs: insights into functions. Nat Rev Genet. 2009;10:155-9.

7. Gibb EA, Vucic EA, Enfield KS, Stewart GL, Lonergan KM, Kennett JY, et al. Human cancer long non-coding RNA transcriptomes. PLoS One. 2011;6:e25915.

8. Castle JC, Armour CD, Lower M, Haynor D, Biery M, Bouzek H, et al. Digital genome-wide ncRNA expression, including SnoRNAs, across 11 human tissues using polyA-neutral amplification. PLoS One. 2010;5:e11779.

9. Wu SC, Kallin EM, Zhang Y. Role of H3K27 methylation in the regulation of IncRNA expression. Cell Res. 2010;20:1109-16.

10. Tsai MC, Manor O, Wan Y, Mosammaparast N, Wang JK, Lan F, et al. Long noncoding RNA as modular scaffold of histone modification complexes. Science. 2010;329:689-93.

11. Kotake Y, Nakagawa T, Kitagawa K, Suzuki S, Liu N, Kitagawa M, et al. Long non-coding RNA ANRIL is required for the PRC2 recruitment to and silencing of p15(INK4B) tumor suppressor gene. Oncogene. 2011;30:1956-62.

12. Gong C, Maquat LE. IncRNAs transactivate STAU1-mediated mRNA decay by duplexing with 3' UTRs via Alu elements. Nature. 2011;470:284-8.

13. Gutschner T, Diederichs S. The hallmarks of cancer: a long non-coding RNA point of view. RNA Biol. 2012;9:703-19.

14. Gibb EA, Brown CJ, Lam WL. The functional role of long non-coding RNA in human carcinomas. Mol Cancer. 2011;10:38.

15. Huarte M, Rinn JL. Large non-coding RNAs: missing links in cancer? Hum Mol Genet. 2010;19:R152-61.

16. Johnson R, Guigo R. The RIDL hypothesis: transposable elements as functional domains of long noncoding RNAs. RNA. 2014;20:959-76.

17. Kapusta A, Kronenberg Z, Lynch VJ, Zhuo X, Ramsay L, Bourque G, et al. Transposable elements are major contributors to the origin, diversification, and regulation of vertebrate long noncoding RNAs. PLoS Genet. 2013;9:e1003470.

18. Kelley D, Rinn J. Transposable elements reveal a stem cell-specific class of long noncoding RNAs. Genome Biol. 2012;13:R107.

19. Cohen CJ, Lock WM, Mager DL. Endogenous retroviral LTRs as promoters for human genes: a critical assessment. Gene. 2009;448:105-14.

20. Lower R, Lower J, Kurth R. The viruses in all of us: characteristics and biological significance of human endogenous retrovirus sequences. Proc Natl Acad Sci U S A. 1996;93:5177-84.

21. Fort A, Hashimoto K, Yamada D, Salimullah M, Keya CA, Saxena A, et al. Deep transcriptome profiling of mammalian stem cells supports a regulatory role for retrotransposons in pluripotency maintenance. Nat Genet. 2014;46:558-66.

22. Faulkner GJ, Kimura Y, Daub CO, Wani S, Plessy C, Irvine KM, et al. The regulated retrotransposon transcriptome of mammalian cells. Nat Genet. 2009:41:563-71.

23. Maksakova IA, Mager DL, Reiss D. Keeping active endogenous retroviral-like elements in check: the epigenetic perspective. Cell Mol Life Sci. 2008;65:3329-47.

24. Stoye JP. Studies of endogenous retroviruses reveal a continuing evolutionary saga. Nat Rev Microbiol. 2012;10:395-406.

25. Kassiotis G. Endogenous retroviruses and the development of cancer. J Immunol. 2014;192:1343-9.

26. Ruprecht K, Mayer J, Sauter M, Roemer K, Mueller-Lantzsch N. Endogenous retroviruses and cancer. Cell Mol Life Sci. 2008;65:3366-82.

27. Stauffer $Y$, Theiler $G$, Sperisen $P$, Lebedev $Y$, Jongeneel CV. Digital expression profiles of human endogenous retroviral families in normal and cancerous tissues. Cancer Immun. 2004;4:2

28. Santoni FA, Guerra J, Luban J. HERV-H RNA is abundant in human embryonic stem cells and a precise marker for pluripotency. Retrovirology. 2012;9:111.

29. The NCBI Sequence Read Archive. http://www.ncbi.n/m.nih.gov/Traces/sra.

30. Wilks C, Cline MS, Weiler E, Diehkans M, Craft B, Martin C, et al. The Cancer Genomics Hub (CGHub): overcoming cancer through the power of torrential data. Database (Oxford). 2014. (https://cghub.ucsc.edu/)

31. Barrett T, Wilhite SE, Ledoux P, Evangelista C, Kim IF, Tomashevsky M, et al. NCBI GEO: archive for functional genomics data sets-update. Nucleic Acids Res; 2013;41:D991-995. (http://www.ncbi.nlm.nih.gov/geo/) 
32. An integrated encyclopedia of DNA elements in the human genome. Nature. 2012;489:57-74. (http://genome.ucsc.edu/ENCODE/).

33. Warren RL, Freeman DJ, Pleasance S, Watson P, Moore RA, Cochrane K, et al. Co-occurrence of anaerobic bacteria in colorectal carcinomas. Microbiome. 2013;1:16.

34. Dobin A, Davis CA, Schlesinger F, Drenkow J, Zaleski C, Jha S, et al. STAR: ultrafast universal RNA-seq aligner. Bioinformatics. 2013;29:15-21.

35. Kim D, Pertea G, Trapnell C, Pimentel H, Kelley R, Salzberg SL. TopHat2: accurate alignment of transcriptomes in the presence of insertions, deletions and gene fusions. Genome Biol. 2013;14:R36.

36. Trapnell C, Williams BA, Pertea G, Mortazavi A, Kwan G, van Baren MJ, et al. Transcript assembly and quantification by RNA-seq reveals unannotated transcripts and isoform switching during cell differentiation. Nat Biotechnol. 2010:28:511-5.

37. Warren RL, Holt RA. Targeted assembly of short sequence reads. PLoS One. 2011;6:e19816.

38. Altschul SF, Gish W, Miller W, Myers EW, Lipman DJ. Basic local alignment search tool. J Mol Biol. 1990;215:403-10.

39. Mortazavi A, Williams BA, McCue K, Schaeffer L, Wold B. Mapping and quantifying mammalian transcriptomes by RNA-seq. Nat Methods. 2008;5:621-8.

40. Castellarin M, Warren RL, Freeman JD, Dreolini L, Krzywinski M, Strauss J, et al. Fusobacterium nucleatum infection is prevalent in human colorectal carcinoma. Genome Res. 2012;22:299-306.

41. Wheeler TJ, Clements J, Eddy SR, Hubley R, Jones TA, Jurka J, et al. Dfam: a database of repetitive DNA based on profile hidden Markov models. Nucleic Acids Res. 2013;41:D70-82.

42. Tamura K, Stecher G, Peterson D, Filipski A, Kumar S. MEGA6: Molecular Evolutionary Genetics Analysis version 6.0. Mol Biol Evol. 2013;30:2725-9.

43. Waterhouse AM, Procter JB, Martin DM, Clamp M, Barton GJ. Jalview Version 2-a multiple sequence alignment editor and analysis workbench. Bioinformatics. 2009;25:1189-91.

44. Sequences Identities and Similarities. http://imed.med.ucm.es/Tools/sias.html.

45. Miller W, Rosenbloom K, Hardison RC, Hou M, Taylor J, Raney B, et al. 28-way vertebrate alignment and conservation track in the UCSC Genome Browser. Genome Res. 2007;17:1797-808.

46. Kuhn RM, Karolchik D, Zweig AS, Trumbower H, Thomas DJ, Thakkapallayil A, et al. The UCSC genome browser database: update 2007. Nucleic Acids Res. 2007:35:D668-73.

47. Kong L, Zhang Y, Ye ZQ, Liu XQ, Zhao SQ, Wei L, et al. CPC: assess the protein-coding potential of transcripts using sequence features and support vector machine. Nucleic Acids Res. 2007;35:W345-9.

48. The Open Reading Frame Finder. http://www.ncbi.nlm.nih.gov/gorf/gorf.html.

49. Banfai B, Jia H, Khatun J, Wood E, Risk B, Gundling Jr WE, et al. Long noncoding RNAs are rarely translated in two human cell lines. Genome Res. 2012;22:1646-57.

50. Ma J, Ward CC, Jungreis I, Slavoff SA, Schwaid AG, Neveu J, et al. Discovery of human sORF-encoded polypeptides (SEPS) in cell lines and tissue. J Proteome Res. 2014;13:1757-65.

51. Slavoff SA, Mitchell AJ, Schwaid AG, Cabili MN, Ma J, Levin JZ, et al. Peptidomic discovery of short open reading frame-encoded peptides in human cells. Nat Chem Biol. 2013;9:59-64.

52. Seo JS, Ju YS, Lee WC, Shin JY, Lee JK, Bleazard T, et al. The transcriptional landscape and mutational profile of lung adenocarcinoma. Genome Res. 2012;22:2109-19.

53. Budczies J, Klauschen F, Sinn BV, Gyorffy B, Schmitt WD, Darb-Esfahani S, et al. Cutoff Finder: a comprehensive and straightforward Web application enabling rapid biomarker cutoff optimization. PLoS One. 2012;7:e51862.

54. Jurka J, Kapitonov W, Klonowski P, Walichiewicz J, Smit AF. Identification of new medium reiteration frequency repeats in the genomes of Primates, Rodentia and Lagomorpha. Genetica. 1996;98:235-47.

55. Fromont-Racine M, Bertrand E, Pictet $R$, Grange $T$. A highly sensitive method for mapping the $5^{\prime}$ termini of mRNAs. Nucleic Acids Res. 1993;21:1683-4.

56. Ha HS, Chung WK, Ahn K, Bae JH, Park SJ, Moon JW, et al. Development of GEBRET: a web-based analysis tool for retroelements in primate genomes. Genes Genom. 2011;33:679-84

57. Ge P, Zhang S. Incorporating phylogenetic-based covarying mutations into RNAalifold for RNA consensus structure prediction. BMC Bioinformatics. 2013;14:142.

58. Subramanian RP, Wildschutte JH, Russo C, Coffin JM. Identification, characterization, and comparative genomic distribution of the HERV-K (HML-2) group of human endogenous retroviruses. Retrovirology. 2011;8:90.
59. Lander ES, Linton LM, Birren B, Nusbaum C, Zody MC, Baldwin J, et al. Initial sequencing and analysis of the human genome. Nature. 2001;409:860-921.

60. Venter JC, Adams MD, Myers EW, Li PW, Mural RJ, Sutton GG, et al. The sequence of the human genome. Science. 2001;291:1304-51.

61. Kapitonov V, Jurka J. The age of Alu subfamilies. J Mol Evol. 1996;42:59-65.

62. Springer MS, Murphy WJ, Eizirik E, O'Brien SJ. Placental mammal diversification and the Cretaceous-Tertiary boundary. Proc Natl Acad Sci U S A. 2003;100:1056-61

63. Marques AC, Ponting CP. Catalogues of mammalian long noncoding RNAs: modest conservation and incompleteness. Genome Biol. 2009;10:R124.

64. Ulitsky I, Bartel DP. lincRNAs: genomics, evolution, and mechanisms. Cell. 2013;154:26-46.

65. Necsulea A, Soumillon M, Warnefors M, Liechti A, Daish T, Zeller U, et al. The evolution of IncRNA repertoires and expression patterns in tetrapods. Nature. 2014;505:635-40.

66. Schuler A, Ghanbarian AT, Hurst LD. Purifying selection on splice-related motifs, not expression level nor RNA folding, explains nearly all constraint on human lincRNAs. Mol Biol Evol. 2014;31:3164-83.

67. Washietl S, Kellis M, Garber M. Evolutionary dynamics and tissue specificity of human long noncoding RNAs in six mammals. Genome Res. 2014;24:616-28.

68. Diederichs $\mathrm{S}$. The four dimensions of noncoding RNA conservation. Trends Genet. 2014;30:121-3.

69. Johnsson P, Lipovich L, Grander D, Morris KV. Evolutionary conservation of long non-coding RNAs; sequence, structure, function. Biochim Biophys Acta. 2014;1840:1063-71.

70. Lamprecht B, Walter K, Kreher S, Kumar R, Hummel M, Lenze D, et al. Derepression of an endogenous long terminal repeat activates the CSF1R proto-oncogene in human lymphoma. Nat Med. 2010;16:571-9. 571p following 579

71. Lock FE, Rebollo R, Miceli-Royer K, Gagnier L, Kuah S, Babaian A, et al Distinct isoform of FABP7 revealed by screening for retroelement-activated genes in diffuse large B-cell lymphoma. Proc Natl Acad Sci U S A. 2014;111:E3534-43.

72. St Laurent G, Shtokalo D, Dong B, Tackett MR, Fan X, Lazorthes S, et al. VlincRNAs controlled by retroviral elements are a hallmark of pluripotency and cancer. Genome Biol. 2013;14:R73.

73. Loewer S, Cabili MN, Guttman M, Loh YH, Thomas K, Park IH, et al. Large intergenic non-coding RNA-RoR modulates reprogramming of human induced pluripotent stem cells. Nat Genet. 2010;42:1113-7.

74. Lu X, Sachs F, Ramsay L, Jacques PE, Goke J, Bourque G, et al. The retrovirus HERVH is a long noncoding RNA required for human embryonic stem cell identity. Nat Struct Mol Biol. 2014;21:423-5.

\section{Submit your next manuscript to BioMed Central and take full advantage of:}

- Convenient online submission

- Thorough peer review

- No space constraints or color figure charges

- Immediate publication on acceptance

- Inclusion in PubMed, CAS, Scopus and Google Scholar

- Research which is freely available for redistribution 